\title{
Cholangiocarcinoma: a site-specific update on the current state of surgical management and multi-modality therapy
}

\author{
Michael K. Turgeon, Shishir K. Maithel \\ Division of Surgical Oncology, Department of Surgery, Winship Cancer Institute, Emory University, Atlanta, GA, USA \\ Contributions: (I) Conception and design: All authors; (II) Administrative support: All authors; (III) Provision of study materials or patients: None; \\ (IV) Collection and assembly of data: None; (V) Data analysis and interpretation: None; (VI) Manuscript writing: All authors; (VII) Final approval of \\ manuscript: All authors. \\ Correspondence to: Shishir K. Maithel, MD, FACS. Division of Surgical Oncology, Department of Surgery, Winship Cancer Institute, Emory \\ University, 1365 Clifton Road, NE, Building B, Suite 4100, Office 4202, Atlanta, GA 30322, USA. Email: smaithe@emory.edu.
}

\begin{abstract}
Biliary tract cancers (BTC) are rare, heterogeneous malignancies that include cholangiocarcinoma and gallbladder cancer (GBC). Cholangiocarcinoma subtypes differ by anatomic location and molecular profile. Currently, resection with lymphadenectomy is the only curative treatment of locally advanced cholangiocarcinoma. Given the high risk of recurrence, multi-modality therapy spanning surgery, chemotherapy, and radiation therapy should be considered. Current data is discordant and there is limited prospective data to support an optimal treatment regimen, though recent studies have demonstrated the utility of adjuvant chemotherapy and chemoradiation in specific settings and patient populations. There is a potential role for neoadjuvant chemotherapy in patients with resectable disease or chemoradiation in select patients with unresectable, locally advanced disease. Randomized clinical trials are necessary to establish the effectiveness of therapies specific to disease sites, especially with the emerging role of immunotherapy and targeted therapy to actionable mutations.
\end{abstract}

Keywords: Cholangiocarcinoma; intrahepatic; hilar; perihilar; surgery; chemotherapy; radiation therapy; chemoradiation

Submitted Jul 22, 2019. Accepted for publication Aug 07, 2019.

doi: $10.21037 /$ cco.2019.08.09

View this article at: http://dx.doi.org/10.21037/cco.2019.08.09

\section{Introduction}

Biliary tract cancers (BTC) encompass a broad spectrum of malignancies which include cholangiocarcinoma and gallbladder cancer (GBC). Based on anatomic location, cholangiocarcinoma is further stratified into intrahepatic cholangiocarcinoma (IHCC), and extrahepatic cholangiocarcinoma (EHCC), which is further subdivided into hilar or perihilar cholangiocarcinoma (PHCC) and distal cholangiocarcinoma (DCC). Notably, tumors that involve the bifurcation of the ducts are known as Klatskin tumors. IHCC comprise approximately $20-30 \%$, PHCC comprise $50 \%$, and DCC comprise $10-30 \%$ of all cholangiocarcinomas $(1,2)$.

Cholangiocarcinoma has differences in growth pattern and can present as mass-forming, periductal-infiltrating, or intraductal. Mass-forming growth patterns are more commonly seen in IHCC, peri-ductal-infiltrating growth in PHCC, while intraductal growth can occur at any location (3).

It is important to note that although these subtypes all arise from the biliary system, there are differences in disease progression, molecular profile, and response to therapy. In this review we focus on the multidisciplinary approach to the management and treatment of cholangiocarcinoma, encompassing surgical and multi-modality therapies.

\section{Epidemiology}

Cholangiocarcinoma is a rare malignancy, accounting for $3 \%$ of all gastrointestinal cancers and $10-15 \%$ of cancers 
of the hepatobiliary system (4). Global incidence of cholangiocarcinoma in 2013 was approximately 186,000 (5). The incidence of IHCC is increasing, while the incidence of EHCC is decreasing, though the latter remains the most common $(6,7)$. Known risk factors for cholangiocarcinoma include cholelithiasis, choledochal cysts, primary sclerosing cholangitis, hepatitis B and C, and liver fluke infections $(8,9)$.

Cholangiocarcinoma is characterized by significant geographic variation. Cholangiocarcinoma is more prevalent in eastern Europe, Asia, and Latin America, while it is more rare in western Europe and the United States (10). IHCC has increased incidence in Western countries in recent years, compared to the incidence of EHCC, which has remained stable (11). These variable incidence rates are likely attributable to genetic and environmental differences.

Cholangiocarcinoma is characterized by early lymph node invasion and distant metastasis. These malignancies often present at an advanced stage and are often inoperable resulting in poor prognosis with a 5 -year overall survival (OS) ranging from $5-15 \%(6,12,13)$. The 5 -year OS survival rates for resected IHCC, PHCC, and DCC are $32.7 \%$, $24.2 \%$, and $39.8 \%$, respectively $(14,15)$. Only approximately $10-30 \%$ of patients are deemed to have resectable disease at the time of diagnosis $(16,17)$.

\section{Distinct sites of disease}

Though cholangiocarcinomas predominantly arise from the epithelial cells of the biliary tree, there is increasing data to support differences in tumor biology that impact clinicopathologic outcomes. Cholangiocarcinomas have discernible gene heterogeneity based on anatomic location and the use of histopathologic and molecular diagnostic techniques suggests variable tumor microenvironment and stroma between the subtypes. Whole genome sequencing performed by the International Cancer Genome Consortium revealed distinct mutations that affect prognosis (18). Broadly, BTCs have clusters of mutations in known oncogenes (KRAS, p53, ERBB2/HER2/NEU) and tumor suppressor genes (SMAD-4, BCL-2, p16, and p53) $(19,20)$.

IHCC uniquely has FGFR1-3 fusion gene mutation rates of $11-17 \%$ and IDH1/2 mutation rates of $5-36 \%(21,22)$. The frequency of KRAS and p53 mutations is $40-50 \%$ and 2.4-44.4\%, respectively (23). EHCC have ERBB2/ HER2/NEU overexpression frequencies of $5-10 \%$, KRAS mutation rates of $8.3-42 \%$, SMAD4 mutation rates $21 \%$, and PIK3CA mutation rates of $7 \%$ (24). IDH1/2 mutations are seen at much lower rates of $0-7 \%$ compared to IHCC. ERBB2/HER2/NEU overexpression is not seen in IHCC (24).

Given the variable molecular landscape of cholangiocarcinoma, there is increased interest in genetic profiling and the application of targeted therapies. Ongoing clinical trials are evaluating the use of FGFR kinase inhibitors (NCT02924376, NCT02150967), IDH1 inhibitors (NCT02989857), and PDL1 immune checkpoint inhibitors (NCT02628067).

\section{Surgical principles}

Currently, resection is the mainstay of treatment for patients who present with resectable disease. The operative strategy is based on the anatomic location of the tumor. For IHCC, a negative-margin resection offers patients the best chance for prolonged survival $(25,26)$. For resectable IHCC, a non-anatomic or anatomic segmental or major hepatic resection with concurrent portal lymphadenectomy is recommended. Extended resections, major vascular resections and reconstructions, and en bloc resections of continguous organs have been reported with acceptable patient outcomes, though the possibility of curative resection must be tailored to each patient's presentation and comorbidities $(27,28)$. Perihilar tumors often pose a technical challenge given their location at the central portion of the liver. As a result, surgical management includes a formal bile duct resection, major hepatic resection with the possibility of a caudate lobe resection, en bloc porta hepatis lymphadenectomy, and a biliary drainage procedure, such as a Roux-en-Y hepaticojejunostomy. The Bismuth-Corlette staging system can aid in operative planning, though this system does not account for vascular or lymph node involvement (29). The surgical management of DCC is classically a pancreatoduodenectomy (Whipple procedure) $(30,31)$.

\section{Determining "resectability"}

Both technical and oncologic considerations must be taken into account when evaluating a patient for resection. First, the patient must be medically fit and able to tolerate a major operation. During the preoperative evaluation, ultrasound, endoscopic ultrasound (EUS), and crosssectional imaging are useful modalities. Specifically, magnetic resonance imaging (MRI) with magnetic resonance cholangiopancreatography (MRCP) helps define the extent of resectability, nodal disease burden, and 
level of biliary obstruction. In select patients, preemptive biliary stenting and/or catheterization can be considered. Endoscopic retrograde cholangiopancreatography (ERCP) with or without cholangioscopy aids in delineating bile duct morphology, particularly the level of strictures. There is the added benefit of obtaining additional diagnostic information via brush cytology or fine needle aspiration (FNA) if a tissue diagnosis is needed prior to resection or the delivery of preoperative therapy. Positron emission tomography (PET) and diagnostic laparoscopy can further define the extent of the tumor burden and assess for disseminated disease to aid in the determination of oncologic appropriateness $(32,33)$. Resection rates for IHCC, PHCC, and DCC were noted to be $56 \%, 60 \%$, and $91 \%$ in a retrospective series of 294 patients over a 23 -year period (25).

Criteria for resection includes the absence of extrahepatic organ invasion, paraceliac and retropancreatic nodal metastasis, distant liver metastasis, disseminated disease, and extensive vascular invasion of the portal vein and hepatic artery (34). Significant vascular involvement of the portal vein or hepatic artery may preclude patients from undergoing surgery as this poses a significant risk factor for disease recurrence and increases postoperative morbidity $(35,36)$. Major vascular resection, however, has been successfully performed in select patients to ensure negative margins and should be discussed in patients with a favorable response to pre-operative therapy $(37,38)$.

\section{Lymphadenectomy}

Lymph node metastasis is a strong negative prognostic indicator $(39,40)$. Portal lymphadenectomy should be performed in all patients with IHCC as up to $30 \%$ of patients will have evidence of lymph node metastasis (41). Lymph node metastasis for IHCC portends reduced survival (24 months for $\mathrm{N} 1,30$ months for $\mathrm{N} 0, \mathrm{P}=0.03$ ) (41). The 8th edition of the AJCC recommends the removal of at least 6 lymph nodes to adequately stage the extent of nodal disease (42). Given the nature of the operations for PHCC and DCC, a regional lymphadenectomy is inherently included in the procedure. Removal of distant lymph nodes, outside the porta hepatis, has not reproducibly been shown to be associated with improved survival.

\section{Future liver remnant (FLR)}

IHCC and PHCC sometimes pose a unique technical dilemma as a major hepatectomy may be indicated. The surgeon must determine if the FLR has adequate inflow, outflow, and biliary drainage. For carefully selected patients with IHCC and PHCC, portal vein embolization (PVE) is a viable option to optimize FLR function.

The liver has a remarkable regenerative capacity for functional recovery after hepatectomy. In patients with healthy, non-cirrhotic livers, $70-80 \%$ of the volume can be removed and still allow for meaningful recovery $(43,44)$. Due to segmental anatomic differences across patients, the implementation of preoperative volumetric analysis aids in predicting FLR function. This can be done using predictive mathematical models or cross-sectional imaging, including CT and 3D CT volumetry $(45,46)$. Though traditionally used in liver transplant patient candidates, functional assessments including the MELD score and the ChildTurcotte-Pugh (CTP) score have been applied to patients being evaluated for oncologic resections as well (47).

PVE is a strategy to maximize the safety of liver surgery and expand the patient population eligible for resection. By occluding the branch of the portal vein on the intended specimen, PVE allows for hypertrophy of the FLR. PVE has been shown to increase the FLR volume an average of $12 \%$ and allow $60 \%$ of patients previously deemed unresectable due to liver capacity limitations to proceed with surgery $(43,48-50)$. Although the application of PVE is not yet the standard of care, prior studies have shown no adverse effect on morbidity and mortality following hepatectomy (51-55). PVE is a worthwhile option for patients where FLR is the only precluding factor for hepatic resection.

\section{Negative margin resection $(\mathrm{R} 0)$}

For resections at any site, an $\mathrm{R} 0$ resection has been shown to be superior to non-operative management and marginpositive resections demonstrating an improvement in OS (56,57). PHCC patients who received an R0 resection and hepatectomy with curative-intent demonstrated improved disease-specific survival (DSS) and OS compared to those undergoing an $\mathrm{R} 1$ resection $(58,59)$. A prospective study in 2012 by Ribero et al. demonstrated a median OS of 39 months and a 5 -year OS rate of $39.8 \%$ for patients with PHCC undergoing an R0 resection (60). For DCC, patients with an R0 resection have a median OS of 48 months, compared to 9 months in patients with an $\mathrm{R} 1$ resection $(\mathrm{P}=0.042)(61)$. The projected ability to achieve an $\mathrm{R} 0$ resection is a major determinant of "technical resectability".

Evaluation of biliary tree involvement is necessary to develop an operative plan for tumor-free margins 
and for potential biliary drainage procedures. Crosssectional imaging allows for visualization of tissue planes. Cholangiography can also be utilized to further characterize bile duct anatomy and to determine the extent of proximal bile duct involvement (62). Historically, for patients who underwent curative intent resections, R0 margins were achieved in approximately $30 \%$ of PHCCs and $50 \%$ of DCCs (63). As the understanding that PHCC required a major hepatectomy in addition to a bile duct resection to achieve an R0 resection became commonly accepted, a 2001 series demonstrated a $78 \%$ rate of $\mathrm{R} 0$ resection for PHCC (59). In a retrospective review of 225 patients with PHCC who underwent resection, the importance of an R0 resection is further emphasized as a microscopic margin was associated with early disease recurrence $(\mathrm{P}=0.04)$ (64). A retrospective review from 2001 to 2012 also compared 96 PHCC patients who received an R0 vs. an R1 resection (65). In this study, a median OS difference (33 vs. 19 months, $\mathrm{P}=0.002$ ) was noted, favoring an $\mathrm{R} 0$ resection. Given the importance of a negative margin, in instances where the margin is positive on intra-operative frozen section analysis, some have advocated for taking an additional resection margin to obtain an R0 margin if technically feasible (66-68). Obtaining negative bile duct margins significantly decrease the probability for locoregional recurrence $(66,69,70)$.

\section{Adjuvant chemotherapy}

Until recently, administration of adjuvant therapy after resection was not standard of care. Furthermore, the optimal regimen had not been well established given conflicting studies that included retrospective series limited by inherent study design and small sample size. Recently completed prospective randomized controlled trials have shaped the consensus guidelines for delivering adjuvant therapy after resection of cholangiocarcinoma.

The initial first-line systemic therapy for advanced disease was established by the ABC-02 trial, a phase III randomized control trial that compared patients with locally advanced or metastatic cholangiocarcinoma, GBC, and ampullary cancer receiving gemcitabine alone to patients receiving gemcitabine plus cisplatin. Gemcitabine plus cisplatin was associated with an increased median OS (11.7 vs. 8 months, $\mathrm{P}<0.001$ ) and progression free survival (8 vs. 5 months, $\mathrm{P}<0001)(71,72)$. It is important to note the treatment arms were comprised of a heterogeneous cohort of patients with biliary tract malignancies.

Given these positive findings with utilizing a doublet regimen consisting of gemcitabine and platinum, the PRODIGE 12-ACCORD 18 (UNICANCER GI) study was conducted in the adjuvant setting, which was a phase III randomized control study of 194 patients with localized BTC (IHCC, PHCC, DCC, and GBC) who underwent a R0 or R1 resection and were randomized to adjuvant chemotherapy with gemcitabine-oxaliplatin (GEMOX) or surveillance alone. Despite a clinically significant difference between groups, no statistically significant difference in OS was noted between the study and control arms (75.8 vs. 50.8 months, $\mathrm{P}=0.74)$, and this trial was deemed a negative trial (73). The Bile Duct Cancer Adjuvant Trial (BCAT) is a randomized phase III trial comprised of patients with resected bile duct cancer comparing adjuvant gemcitabine vs. observation (74). The study determined there was no significant difference in median OS (62.3 vs. 63.8 months, $\mathrm{P}=0.96$ ) or RFS (36 vs. 39.9 months, $\mathrm{P}=0.69$ ) between the adjuvant gemcitabine and observation cohorts. Notably, the study was under-powered as it did not accrue the planned number of patients.

The BILCAP trial was the first randomized controlled, phase III multicenter trial of 447 patients with IHCC, PHCC, muscle-invasive GBC, and lower bile duct cholangiocarcinoma that demonstrated an OS benefit of adjuvant chemotherapy with capecitabine over surveillance alone. Though there was no significant difference in OS in the intent-to-treat analysis, patients who received adjuvant capecitabine in the per-protocol analysis had an increased median OS of 51 months compared to 36 months in the placebo observation group $(\mathrm{P}=0.028)$ (75). The BILCAP trial has largely informed current consensus guidelines favoring adjuvant chemotherapy with 6 months of capecitabine for resected biliary tract malignancies.

There are other ongoing trials to further elucidate the optimal adjuvant chemotherapy regimen. The Japanese S-1 vs. placebo JCOG1202 is a randomized phase III trial of 350 patients with biliary tract adenocarcinoma or adenosquamous carcinoma (IHCC, EHCC, GBC, or ampulla of Vater cancer) comparing adjuvant S-1 therapy $v s$. observation alone in resected BTC with a primary end-point of median OS (UMIN000011688) (76). The German Adjuvant Chemotherapy with Gemcitabine and Cisplatin Compared to Standard of Care after Curative Intent Resection of Biliary Tract Cancer (ACTICCA-1) phase III randomized controlled trial includes 781 patients diagnosed with IHCC, PHCC, EHCC, and muscle invasive gallbladder carcinoma comparing adjuvant cisplatin and gemcitabine to standard of care (curative intent resection) 
with the endpoint of DFS (NCT02170090). The standardof-care arm was revised to include adjuvant capecitabine in light of the data from the BILCAP trial (77).

The ASCO Clinical Practice Guideline offers treatment recommendations for patients with resected BTCs. The current standard of care is to offer adjuvant capecitabine chemotherapy for 6 months based on the data in the BILCAP trial $(75,78)$. Continued patient enrollment in clinical trials is highly encouraged to further define the optimal regimen.

\section{Adjuvant radiation therapy}

There is limited data available to support adjuvant radiation in IHCC after resection (12). For patients with EHCC, including both PHCC and DCC, the selective use of adjuvant chemoradiation may be warranted, particularly in the setting of an R1 resection $(79,80)$.

A retrospective study by Shinohara and colleagues demonstrated the potential utility of adjuvant radiation therapy for IHCC. Patients who underwent surgery and received adjuvant radiation therapy had a median OS of 11 months, compared to 6 months for patients that underwent surgery alone, and 7 months for patients that received radiation alone as their primary therapy $(\mathrm{P}=0.013)$ (79). In a retrospective review of 92 patients with unresectable and advanced IHCC, Kim et al. demonstrated that patients who received chemoradiation had improved OS and PFS compared to patients that received chemotherapy alone (OS 9.1 vs. 6.2 months, $\mathrm{P}<0.05$ and PFS 4.3 vs. 1.9 months, $\mathrm{P}=0.001$ ) (81). Importantly, this study included patients with locally advanced disease that did not undergo resection. There is a need for randomized control trials to determine the role of chemoradiation for locally advanced, non-resectable IHCC compared to chemotherapy alone. Unfortunately, one such trial recently closed due to poor accrual.

A 2007 study of 75 patients with PHCC demonstrated that patients who received adjuvant radiation therapy after an R1 or R2 resection had improved OS compared to observation alone $(82,83)$. There was no association between radiation and OS in those who achieved an $\mathrm{R} 0$ resection. Accordingly, in instances where there are positive margins after surgery for patients with EHCC, there may be a role for salvage radiation therapy to address residual disease.

A 2003 retrospective review by Heron et al. demonstrated increased median OS for 221 patients with proximal EHCC who underwent surgery and received adjuvant radiation, compared to radiation alone ( 24 vs. 13 months, $\mathrm{P}=0.007$ ), again demonstrating the importance of resection for this disease (84). SWOG0809 is a prospective, phase II, singlearm trial of 79 patents with resected EHCC and GBC who received adjuvant capecitabine and gemcitabine followed by concurrent radiation and capecitabine. Median OS was 35 months (85). Given the single-arm study design, it is unclear whether this survival benefit is from chemotherapy or radiation therapy. Importantly, patients with an R1 resection had similar survival as those with an $\mathrm{R} 0$ resection, suggesting the potential utility of radiation in margin positive resections.

There are no prospective trials investigating the role of adjuvant therapy specifically for DCC. Current retrospective data is mixed and is often extrapolated from pancreatic cancer studies. At this time, there is a lack of strong data to support the use of adjuvant radiation therapy for DCC, unless considering the circumstance of an R1 resection.

Current ASCO clinical practice guidelines are largely based on SWOG0809, recommending chemoradiation for patients with hilar cholangiocarcinoma and DCC with positive surgical margins (R1 or R2 resection) (78). Given current study limitations and paucity of definitive data, further investigation is needed to determine the utility of adjuvant radiation or chemoradiation for locoregional control, particularly for patients with IHCC.

\section{Neoadjuvant therapy}

There are no formal recommendations for neoadjuvant therapy in the NCCN guidelines or ASCO clinical practice guidelines. Given the overall dismal outcomes for cholangiocarcinoma and similarity to pancreas cancer, it inherently follows that efforts should be directed at determining the role of neoadjuvant therapy prior to resection. The heterogeneity and unique challenges associated with each disease site mandate a separate discussion for each anatomic location of cholangiocarcinoma.

\section{IHCC}

Le Roy et al. conducted a retrospective study of 186 patients between 2000 and 2013 with resectable IHCC comparing neoadjuvant chemotherapy, upfront surgery, and chemotherapy alone (86). Median OS was 24.1 months in the neoadjuvant chemotherapy and surgery cohort, 
25.7 months in the surgery-only cohort, and 7.8 months in the chemotherapy-only cohort. There was no significant difference between the neoadjuvant chemotherapy and the upfront surgery group. There may, however, be a role for neoadjuvant treatment in patients with resectable but oncologically high-risk IHCC including large lesions $>5$ $\mathrm{cm}$, multifocal tumors or satellite lesions confined to the same lobe of the liver as the dominant lesion, the presence of major vascular invasion, and suspicious or involved regional lymph nodes ( $\mathrm{N} 1$ disease). There is an ongoing phase II trial to determine the feasibility of neoadjuvant gemcitabine, cisplatin, and abraxane (nab-paclitaxel) in this patient population with primary outcomes of preoperative/operative therapy completion and incidence of adverse events (NCT03579771). Others have suggested that there may be an advantage from chemoradiation to down-stage the tumor burden and increase the likelihood of resectability in patients who present with locally advanced unresectable disease $(87,88)$. The Le Roy et al. study discussed above also demonstrated $50 \%$ conversion from unresectable to resectable disease with the use of neoadjuvant chemotherapy, supporting its use alone without radiation.

\section{PHCC}

A definitive tissue diagnosis for PHCC is often difficult to obtain prior to resection, thus making delivering neoadjuvant therapy a challenging therapeutic strategy. The armamentarium to make a diagnosis includes cholangiography (either endoscopic or percutaneous), spy glass cholangioscopy, and fluorescence in situ hybridization (FISH). Brushings via cholangiography allows for tissue sampling, though the sensitivity is highly variable, ranging from $20-50 \%$ (89-91). Spy glass cholangioscopy is another modality that provides direct visualization of the biliary system. Cholangioscopy has increased sensitivity and specificity (90\% and $95.8 \%$, respectively) when compared to ERCP $(92,93)$. FISH has been shown to increase the sensitivity of cholangiography to $35-60 \%$ as well (94). A retrospective review from 1983 to 1996 of 81 patients with EHCC did not show a mean OS difference in patients who received neoadjuvant chemoradiation, post-operative chemoradiation, post-operative radiation therapy, or surgery alone, though it is worth noting $100 \%$ of the patients who received neoadjuvant chemoradiation underwent an $\mathrm{R} 0$ resection $(\mathrm{P}<0.001)$. A more recent retrospective review of 57 patients with $\mathrm{PHCC}$ comparing neoadjuvant radiation to surgery alone had similar findings (95). Despite negative margin resections, however, there was no statistically significant difference in median DFS or OS $(\mathrm{P}=0.91$, $\mathrm{P}=0.26$, respectively).

Neoadjuvant radiation therapy confers unique challenges during the operation itself. The creation of new anastomoses with tiny, irradiated ducts may prove technically difficult. This may lead to an increased bile leak rate. A retrospective review of 28 patients with locally advanced gallbladder carcinoma who received neoadjuvant chemoradiation demonstrated a leak rate of $43 \%$ (96). At this time, there is no reported data on bile leak rates for patients with cholangiocarcinoma who received neoadjuvant radiation or chemoradiation $(97,98)$. The utilization of liver transplantation for patients with unresectable PHCC provides the largest experience with neoadjuvant therapy for cholangiocarcinoma. Patients who meet the strict inclusion criteria for transplant are treated with combination neoadjuvant chemoradiation prior to liver transplantation. During transplant, the entire biliary apparatus is removed and replaced, thus eliminating the risk of biliary reconstruction after radiation seen with resection. A protocol developed at the Mayo Clinic highlights the efficacy of neoadjuvant chemoradiation and liver transplantation $v s$. resection for patients with Klatskin tumors. In their original report, 38 patients received a liver transplant and 54 patients underwent a hepatic resection. The 5 -year OS rate for the transplant cohort was $82 \%$, compared to $21 \%$ in the resection group $(\mathrm{P}=0.022)$ (99). A retrospective study of 287 patients by Darwish Murad et al. had a RFS of $78 \%$ and $65 \%$ at 2 and 5 years $(\mathrm{P}<0.001)$, respectively (100). A prospective study by Loveday and colleagues demonstrated a 2-year post-transplant OS of $55.6 \%$ in 6 patients (101). Based on these data, neoadjuvant chemoradiation prior to liver transplantation should be considered in patients with unresectable PHCC that meet the strict inclusion criteria.

\section{$D C C$}

It is difficult to differentiate DCC from pancreatic ductal adenocarcinoma (PDAC) in the head of the pancreas preoperatively given the often-indistinguishable imaging findings. In addition, there are no known proteins that are differentially expressed by malignant cells of the biliary tract compared to pancreas cancer. Consequently, there is no immunohistochemical (IHC) marker for biliary epithelium. Though cytokeratin-7 is suggestive of pancreato-biliary 
origin, it is not specific to cholangiocarcinoma (102). Gross evaluation of the resection specimen is needed for a definitive diagnosis. Thus, developing neoadjuvant protocols specifically for DCC is challenging because of the inability to accurately identify the study population in the preoperative setting. In a limited retrospective analysis by McMasters et al., 9 patients from 1983 to 1996 received neoadjuvant chemoradiation for EHCC (5 PHCC, 4 DCC) followed by surgery. An R0 resection margin was obtained at a rate of $100 \%$ for patients who received neoadjuvant therapy, compared to $54 \%$ in patients who did not $(\mathrm{P}<0.01)(98)$. These data support the role of neoadjuvant therapy in increasing the likelihood of achieving an R0 resection. Larger prospective studies are needed, but are limited by the logistical constraints outlined above.

\section{Conclusions}

BTC is comprised of a heterogeneous group of malignancies spanning cholangiocarcinoma and GBC. Subtypes of cholangiocarcinoma differ in anatomic location and molecular profiles, thus presenting unique opportunities for actionable targets. Localized cholangiocarcinoma is amenable to curative-intent resection and should be accompanied with a regional lymphadenectomy. Given the high risk of recurrence, multi-modality therapy encompassing surgery, chemotherapy, and radiation therapy should be discussed in the context of a multidisciplinary team. There is limited prospective data at present to define the optimal therapy for cholangiocarcinoma. Despite this, recent trials and pooled data support the use of adjuvant capecitabine after resection of biliary tract malignancies. There may be a role for the selective use of adjuvant radiation in patients with positive margins. For patients with high-risk features, neoadjuvant chemotherapy should be considered, but needs to be studied. Additional prospective, randomized control trials are needed to ascertain the effectiveness of therapies specific to the different disease sites. There is an ongoing need for continued cooperation and international collaboration to continue to improve patient outcomes. The future is promising with the expanded focus on clinical trial enrollment, particularly with the emerging role of precision medicine and immunotherapy.

\section{Acknowledgments}

None.

\section{Footnote}

Conflicts of Interest: The authors have no conflicts of interest to declare.

Ethical Statement: The authors are accountable for all aspects of the work in ensuring that questions related to the accuracy or integrity of any part of the work are appropriately investigated and resolved.

\section{References}

1. DeOliveira ML, Cunningham SC, Cameron JL, et al. Cholangiocarcinoma: thirty-one-year experience with 564 patients at a single institution. Ann Surg 2007;245:755-62.

2. Razumilava N, Gores GJ. Cholangiocarcinoma. Lancet 2014;383:2168-79.

3. Lim JH. Cholangiocarcinoma: morphologic classification according to growth pattern and imaging findings. AJR Am J Roentgenol 2003;181:819-27.

4. Vauthey JN, Blumgart LH. Recent advances in the management of cholangiocarcinomas. Semin Liver Dis 1994;14:109-14.

5. Fitzmaurice C, Dicker D, Pain A, et al. The Global Burden of Cancer 2013. JAMA Oncology 2015;1:505.

6. Shaib Y, El-Serag HB. The epidemiology of cholangiocarcinoma. Semin Liver Dis 2004;24:115-25.

7. Endo I, Gonen M, Yopp AC, et al. Intrahepatic cholangiocarcinoma: rising frequency, improved survival, and determinants of outcome after resection. Ann Surg 2008;248:84-96.

8. Lee CH, Chang CJ, Lin YJ, et al. Viral hepatitis-associated intrahepatic cholangiocarcinoma shares common disease processes with hepatocellular carcinoma. Br J Cancer 2009; 100:1765-70.

9. El-Serag HB, Engels EA, Landgren O, et al. Risk of hepatobiliary and pancreatic cancers after hepatitis $\mathrm{C}$ virus infection: A population-based study of U.S. veterans. Hepatology 2009;49:116-23.

10. Randi G, Malvezzi M, Levi F, et al. Epidemiology of biliary tract cancers: an update. Ann Oncol 2009;20:146-59.

11. Saha SK, Zhu AX, Fuchs CS, et al. Forty-Year Trends in Cholangiocarcinoma Incidence in the U.S.: Intrahepatic Disease on the Rise. Oncologist 2016;21:594-9.

12. Horgan AM, Amir E, Walter T, et al. Adjuvant therapy in the treatment of biliary tract cancer: a systematic review and meta-analysis. J Clin Oncol 2012;30:1934-40. 
13. Patel T. Cholangiocarcinoma. Nat Clin Pract Gastroenterol Hepatol 2006;3:33-42.

14. Ishihara S, Horiguchi A, Miyakawa S, et al. Biliary tract cancer registry in Japan from 2008 to 2013. J Hepatobiliary Pancreat Sci 2016;23:149-57.

15. Kudo M, Izumi N, Ichida T, et al. Report of the 19th follow-up survey of primary liver cancer in Japan. Hepatol Res 2016;46:372-90.

16. Khan SA, Davidson BR, Goldin RD, et al. Guidelines for the diagnosis and treatment of cholangiocarcinoma: an update. Gut 2012;61:1657-69.

17. Newcomb PA, Storer BE, Morimoto LM, et al. Long-term efficacy of sigmoidoscopy in the reduction of colorectal cancer incidence. J Natl Cancer Inst 2003;95:622-5.

18. Bagante F, Ruzzenente A, Conci S, et al. Patterns of gene mutations in bile duct cancers: is it time to overcome the anatomical classification? HPB (Oxford) 2019;21:1648-55.

19. Reeves ME, Dematteo RP. Genes and viruses in hepatobiliary neoplasia. Semin Surg Oncol 2000;19:84-93.

20. Jusakul A, Cutcutache I, Yong CH, et al. WholeGenome and Epigenomic Landscapes of Etiologically Distinct Subtypes of Cholangiocarcinoma. Cancer Discov 2017;7:1116-35.

21. Valle JW, Lamarca A, Goyal L, et al. New Horizons for Precision Medicine in Biliary Tract Cancers. Cancer Discov 2017;7:943-62.

22. Borger DR, Tanabe KK, Fan KC, et al. Frequent mutation of isocitrate dehydrogenase (IDH) 1 and IDH2 in cholangiocarcinoma identified through broad-based tumor genotyping. Oncologist 2012;17:72-9.

23. Hezel AF, Deshpande V, Zhu AX. Genetics of biliary tract cancers and emerging targeted therapies. J Clin Oncol 2010;28:3531-40.

24. Nakazawa K, Dobashi Y, Suzuki S, et al. Amplification and overexpression of c-erbB-2, epidermal growth factor receptor, and c-met in biliary tract cancers. J Pathol 2005;206:356-65.

25. Nakeeb A, Pitt HA, Sohn TA, et al. Cholangiocarcinoma. A spectrum of intrahepatic, perihilar, and distal tumors. Ann Surg 1996;224:463-73; discussion 473-5.

26. Cherqui D, Tantawi B, Alon R, et al. Intrahepatic cholangiocarcinoma. Results of aggressive surgical management. Arch Surg 1995;130:1073-8.

27. Lang H, Sotiropoulos GC, Fruhauf NR, et al. Extended hepatectomy for intrahepatic cholangiocellular carcinoma (ICC): when is it worthwhile? Single center experience with 27 resections in 50 patients over a 5 -year period. Ann Surg 2005;241:134-43.
28. Puhalla H, Schuell B, Pokorny H, et al. Treatment and outcome of intrahepatic cholangiocellular carcinoma. Am J Surg 2005;189:173-7.

29. Ashai N, Prasad P, Rajdev L. Multimodality Management of Localized Biliary Cancer. Curr Treat Options Oncol 2019;20:58.

30. Banales JM, Cardinale V, Carpino G, et al. Cholangiocarcinoma: current knowledge and future perspectives consensus statement from the European Network for the Study of Cholangiocarcinoma (ENSCCA). Nat Rev Gastroenterol Hepatol 2016;13:261-80.

31. Loehrer AP, House MG, Nakeeb A, et al. Cholangiocarcinoma: are North American surgical outcomes optimal? J Am Coll Surg 2013;216:192-200.

32. Corvera CU, Blumgart LH, Akhurst T, et al. 18F-fluorodeoxyglucose positron emission tomography influences management decisions in patients with biliary cancer. J Am Coll Surg 2008;206:57-65.

33. Connor S, Barron E, Wigmore SJ, et al. The utility of laparoscopic assessment in the preoperative staging of suspected hilar cholangiocarcinoma. J Gastrointest Surg 2005;9:476-80.

34. Rajagopalan V, Daines WP, Grossbard ML, et al. Gallbladder and biliary tract carcinoma: A comprehensive update, Part 1. Oncology (Williston Park) 2004;18:889-96.

35. Wang Y, Li J, Xia Y, et al. Prognostic nomogram for intrahepatic cholangiocarcinoma after partial hepatectomy. J Clin Oncol 2013;31:1188-95.

36. Hyder O, Marques H, Pulitano C, et al. A nomogram to predict long-term survival after resection for intrahepatic cholangiocarcinoma: an Eastern and Western experience. JAMA Surg 2014;149:432-8.

37. Pawlik TM, Poon RT, Abdalla EK, et al. Hepatectomy for hepatocellular carcinoma with major portal or hepatic vein invasion: results of a multicenter study. Surgery 2005;137:403-10.

38. Spolverato G, Ejaz A, Kim Y, et al. Tumor size predicts vascular invasion and histologic grade among patients undergoing resection of intrahepatic cholangiocarcinoma. J Gastrointest Surg 2014;18:1284-91.

39. Kiriyama M, Ebata T, Aoba T, et al. Prognostic impact of lymph node metastasis in distal cholangiocarcinoma. Br J Surg 2015;102:399-406.

40. Hyder O, Hatzaras I, Sotiropoulos GC, et al. Recurrence after operative management of intrahepatic cholangiocarcinoma. Surgery 2013;153:811-8.

41. de Jong MC, Nathan H, Sotiropoulos GC, et al. Intrahepatic cholangiocarcinoma: an international multi- 
institutional analysis of prognostic factors and lymph node assessment. J Clin Oncol 2011;29:3140-5.

42. Amin MB, Greene FL, Edge SB, et al. The Eighth Edition AJCC Cancer Staging Manual: Continuing to build a bridge from a population-based to a more "personalized" approach to cancer staging. CA Cancer J Clin 2017;67:93-9.

43. Abdalla EK, Barnett CC, Doherty D, et al. Extended hepatectomy in patients with hepatobiliary malignancies with and without preoperative portal vein embolization. Arch Surg 2002;137:675-80; discussion 680-1.

44. Vauthey JN, Pawlik TM, Abdalla EK, et al. Is extended hepatectomy for hepatobiliary malignancy justified? Ann Surg 2004;239:722-30; discussion 730-2.

45. Saito S, Yamanaka J, Miura K, et al. A novel 3D hepatectomy simulation based on liver circulation: application to liver resection and transplantation. Hepatology 2005;41:1297-304.

46. Yamanaka J, Saito S, Fujimoto J. Impact of preoperative planning using virtual segmental volumetry on liver resection for hepatocellular carcinoma. World J Surg 2007;31:1249-55.

47. Cucchetti A, Ercolani G, Vivarelli M, et al. Impact of model for end-stage liver disease (MELD) score on prognosis after hepatectomy for hepatocellular carcinoma on cirrhosis. Liver Transpl 2006;12:966-71.

48. Ribero D, Abdalla EK, Madoff DC, et al. Portal vein embolization before major hepatectomy and its effects on regeneration, resectability and outcome. Br J Surg 2007;94:1386-94.

49. Shindoh J, Truty MJ, Aloia TA, et al. Kinetic growth rate after portal vein embolization predicts posthepatectomy outcomes: toward zero liver-related mortality in patients with colorectal liver metastases and small future liver remnant. J Am Coll Surg 2013;216:201-9.

50. Lam VW, Laurence JM, Johnston E, et al. A systematic review of two-stage hepatectomy in patients with initially unresectable colorectal liver metastases. HPB (Oxford) 2013;15:483-91.

51. Bennett JJ, Blumgart LH. Assessment of hepatic reserve prior to hepatic resection. J Hepatobiliary Pancreat Surg 2005;12:10-5.

52. Farges O, Belghiti J, Kianmanesh R, et al. Portal vein embolization before right hepatectomy: prospective clinical trial. Ann Surg 2003;237:208-17.

53. Ethun CG, Maithel SK. Determination of Resectability. Surg Clin North Am 2016;96:163-81.

54. Cillo U, Fondevila C, Donadon M, et al. Surgery for cholangiocarcinoma. Liver Int 2019;39 Suppl 1:143-55.

55. Biggemann L, Uhlig J, Streit U, et al. Future liver remnant growth after various portal vein embolization regimens: a quantitative comparison. Minim Invasive Ther Allied Technol 2019. [Epub ahead of print].

56. Konstadoulakis MM, Roayaie S, Gomatos IP, et al. Fifteen-year, single-center experience with the surgical management of intrahepatic cholangiocarcinoma: operative results and long-term outcome. Surgery 2008;143:366-74.

57. Wu L, Tsilimigras DI, Paredes AZ, et al. Trends in the Incidence, Treatment and Outcomes of Patients with Intrahepatic Cholangiocarcinoma in the USA: Facility Type is Associated with Margin Status, Use of Lymphadenectomy and Overall Survival. World J Surg 2019;43:1777-87.

58. Rocha FG, Matsuo K, Blumgart LH, et al. Hilar cholangiocarcinoma: the Memorial Sloan-Kettering Cancer Center experience. J Hepatobiliary Pancreat Sci 2010;17:490-6.

59. Jarnagin WR, Fong Y, DeMatteo RP, et al. Staging, resectability, and outcome in 225 patients with hilar cholangiocarcinoma. Ann Surg 2001;234:507-17; discussion 517-9.

60. Ribero D, Pinna AD, Guglielmi A, et al. Surgical Approach for Long-term Survival of Patients With Intrahepatic Cholangiocarcinoma: A Multi-institutional Analysis of 434 Patients. Arch Surg 2012;147:1107-13.

61. Chua TC, Mittal A, Arena J, et al. Resection margin influences survival after pancreatoduodenectomy for distal cholangiocarcinoma. Am J Surg 2017;213:1072-6.

62. Sano T, Shimada K, Sakamoto Y, et al. One hundred two consecutive hepatobiliary resections for perihilar cholangiocarcinoma with zero mortality. Ann Surg 2006;244:240-7.

63. Burke EC, Jarnagin WR, Hochwald SN, et al. Hilar Cholangiocarcinoma: patterns of spread, the importance of hepatic resection for curative operation, and a presurgical clinical staging system. Ann Surg 1998;228:385-94.

64. Zhang XF, Beal EW, Chakedis J, et al. Defining Early Recurrence of Hilar Cholangiocarcinoma After Curativeintent Surgery: A Multi-institutional Study from the US Extrahepatic Biliary Malignancy Consortium. World J Surg 2018;42:2919-29.

65. Yoo T, Park SJ, Han SS, et al. Proximal Resection Margins: More Prognostic than Distal Resection Margins in Patients Undergoing Hilar Cholangiocarcinoma Resection. Cancer Res Treat 2018;50:1106-13.

66. Seyama Y, Kubota K, Sano K, et al. Long-term 
outcome of extended hemihepatectomy for hilar bile duct cancer with no mortality and high survival rate. Ann Surg 2003;238:73-83.

67. Sakamoto E, Nimura Y, Hayakawa N, et al. The pattern of infiltration at the proximal border of hilar bile duct carcinoma: a histologic analysis of 62 resected cases. Ann Surg 1998;227:405-11.

68. Endo I, House MG, Klimstra DS, et al. Clinical significance of intraoperative bile duct margin assessment for hilar cholangiocarcinoma. Ann Surg Oncol 2008;15:2104-12.

69. Park Y, Hwang DW, Kim JH, et al. Prognostic comparison of the longitudinal margin status in distal bile duct cancer: $\mathrm{R} 0$ on first bile duct resection versus $\mathrm{R} 0$ after additional resection. J Hepatobiliary Pancreat Sci 2019;26:169-78.

70. Zhang XF, Squires MH, 3rd, Bagante F, et al. The Impact of Intraoperative Re-Resection of a Positive Bile Duct Margin on Clinical Outcomes for Hilar Cholangiocarcinoma. Ann Surg Oncol 2018;25:1140-9.

71. Valle J, Wasan H, Palmer DH, et al. Cisplatin plus gemcitabine versus gemcitabine for biliary tract cancer. $\mathrm{N}$ Engl J Med 2010;362:1273-81.

72. Lischalk JW, Repka MC, Unger K. Radiation therapy for hepatobiliary malignancies. J Gastrointest Oncol 2017;8:279-92.

73. Edeline J, Benabdelghani M, Bertaut A, et al. Gemcitabine and Oxaliplatin Chemotherapy or Surveillance in Resected Biliary Tract Cancer (PRODIGE 12-ACCORD 18-UNICANCER GI): A Randomized Phase III Study. J Clin Oncol 2019;37:658-67.

74. Ebata T, Hirano S, Konishi M, et al. Randomized clinical trial of adjuvant gemcitabine chemotherapy versus observation in resected bile duct cancer. Br J Surg 2018;105:192-202.

75. Primrose JN, Fox RP, Palmer DH, et al. Capecitabine compared with observation in resected biliary tract cancer (BILCAP): a randomised, controlled, multicentre, phase 3 study. Lancet Oncol 2019;20:663-73.

76. Nakachi K, Konishi M, Ikeda M, et al. A randomized Phase III trial of adjuvant S-1 therapy vs. observation alone in resected biliary tract cancer: Japan Clinical Oncology Group Study (JCOG1202, ASCOT). Jpn J Clin Oncol 2018;48:392-5.

77. Stein A, Arnold D, Bridgewater J, et al. Adjuvant chemotherapy with gemcitabine and cisplatin compared to observation after curative intent resection of cholangiocarcinoma and muscle invasive gallbladder carcinoma (ACTICCA-1 trial) - a randomized, multidisciplinary, multinational phase III trial. BMC Cancer 2015;15:564.

78. Shroff RT, Kennedy EB, Bachini M, et al. Adjuvant Therapy for Resected Biliary Tract Cancer: ASCO Clinical Practice Guideline. J Clin Oncol 2019;37:1015-27.

79. Shinohara ET, Mitra N, Guo M, et al. Radiation therapy is associated with improved survival in the adjuvant and definitive treatment of intrahepatic cholangiocarcinoma. Int J Radiat Oncol Biol Phys 2008;72:1495-501.

80. Pitt HA, Nakeeb A, Abrams RA, et al. Perihilar cholangiocarcinoma. Postoperative radiotherapy does not improve survival. Ann Surg 1995;221:788-97; discussion 797-8.

81. Kim YI, Park JW, Kim BH, et al. Outcomes of concurrent chemoradiotherapy versus chemotherapy alone for advanced-stage unresectable intrahepatic cholangiocarcinoma. Radiat Oncol 2013;8:292.

82. Cheng Q, Luo X, Zhang B, et al. Predictive factors for prognosis of hilar cholangiocarcinoma: postresection radiotherapy improves survival. Eur J Surg Oncol 2007;33:202-7.

83. Kim E, Kim YJ, Kim K, et al. Salvage radiotherapy for locoregionally recurrent extrahepatic bile duct cancer after radical surgery. Br J Radiol 2017;90:20170308.

84. Heron DE, Stein DE, Eschelman DJ, et al. Cholangiocarcinoma: the impact of tumor location and treatment strategy on outcome. Am J Clin Oncol 2003;26:422-8.

85. Ben-Josef E, Guthrie KA, El-Khoueiry AB, et al. SWOG S0809: A Phase II Intergroup Trial of Adjuvant Capecitabine and Gemcitabine Followed by Radiotherapy and Concurrent Capecitabine in Extrahepatic Cholangiocarcinoma and Gallbladder Carcinoma. J Clin Oncol 2015;33:2617-22.

86. Le Roy B, Gelli M, Pittau G, et al. Neoadjuvant chemotherapy for initially unresectable intrahepatic cholangiocarcinoma. Br J Surg 2018;105:839-47.

87. Chun YS, Pawlik TM, Vauthey JN. 8th Edition of the AJCC Cancer Staging Manual: Pancreas and Hepatobiliary Cancers. Ann Surg Oncol 2018;25:845-7.

88. Hong SM, Pawlik TM, Cho H, et al. Depth of tumor invasion better predicts prognosis than the current American Joint Committee on Cancer T classification for distal bile duct carcinoma. Surgery 2009;146:250-7.

89. Farrell RJ, Jain AK, Brandwein SL, et al. The combination of stricture dilation, endoscopic needle aspiration, and biliary brushings significantly improves diagnostic yield from malignant bile duct strictures. Gastrointest Endosc 
2001;54:587-94.

90. Fogel EL, deBellis M, McHenry L, et al. Effectiveness of a new long cytology brush in the evaluation of malignant biliary obstruction: a prospective study. Gastrointest Endosc 2006;63:71-7.

91. Smoczynski M, Jablonska A, Matyskiel A, et al. Routine brush cytology and fluorescence in situ hybridization for assessment of pancreatobiliary strictures. Gastrointest Endosc 2012;75:65-73.

92. Dimas ID, Fragaki M, Vardas E, et al. Digital cholangioscopy (Spyglass) in the diagnosis of cholangiocarcinoma. Ann Gastroenterol 2017;30:253.

93. Navaneethan U, Hasan MK, Kommaraju K, et al. Digital, single-operator cholangiopancreatoscopy in the diagnosis and management of pancreatobiliary disorders: a multicenter clinical experience (with video). Gastrointest Endosc 2016;84:649-55.

94. Brooks C, Gausman V, Kokoy-Mondragon C, et al. Role of Fluorescent In Situ Hybridization, Cholangioscopic Biopsies, and EUS-FNA in the Evaluation of Biliary Strictures. Dig Dis Sci 2018;63:636-44.

95. Jung JH, Lee HJ, Lee HS, et al. Benefit of neoadjuvant concurrent chemoradiotherapy for locally advanced perihilar cholangiocarcinoma. World J Gastroenterol 2017;23:3301-8.

96. Engineer R, Goel M, Chopra S, et al. Neoadjuvant

Cite this article as: Turgeon MK, Maithel SK. Cholangiocarcinoma: a site-specific update on the current state of surgical management and multi-modality therapy. Chin Clin Oncol 2020;9(1):4. doi: 10.21037/сco.2019.08.09
Chemoradiation Followed by Surgery for Locally Advanced Gallbladder Cancers: A New Paradigm. Ann Surg Oncol 2016;23:3009-15.

97. Nelson JW, Ghafoori AP, Willett CG, et al. Concurrent chemoradiotherapy in resected extrahepatic cholangiocarcinoma. Int J Radiat Oncol Biol Phys 2009;73:148-53.

98. McMasters KM, Tuttle TM, Leach SD, et al. Neoadjuvant chemoradiation for extrahepatic cholangiocarcinoma. Am J Surg 1997;174:605-8; discussion 608-9.

99. Rea DJ, Heimbach JK, Rosen CB, et al. Liver transplantation with neoadjuvant chemoradiation is more effective than resection for hilar cholangiocarcinoma. Ann Surg 2005;242:451-8; discussion 458-61.

100.Darwish Murad S, Kim WR, Harnois DM, et al. Efficacy of neoadjuvant chemoradiation, followed by liver transplantation, for perihilar cholangiocarcinoma at 12 US centers. Gastroenterology 2012;143:88-98.e3; quiz e14.

101.Loveday BPT, Knox JJ, Dawson LA, et al. Neoadjuvant hyperfractionated chemoradiation and liver transplantation for unresectable perihilar cholangiocarcinoma in Canada. J Surg Oncol 2018;117:213-9.

102. Chu P, Wu E, Weiss LM. Cytokeratin 7 and cytokeratin 20 expression in epithelial neoplasms: a survey of 435 cases. Mod Pathol 2000;13:962-72. 